\title{
La revisión por pares pospublicación: otro control de calidad del registro científico en biomedicina
}

\author{
Mirna Martínez-Saucedo, ${ }^{1}$ Samara Téllez-Camacho, ${ }^{2}$ Guillermo Aquino-Jarquín, ${ }^{1}$ Rocío Sánchez-Urbina ${ }^{3}$ \\ y Javier T. Granados-Riverón ${ }^{1 *}$ \\ ${ }^{1}$ Secretaría de Salud, Hospital Infantil de México "Federico Gómez", Laboratorio de Investigación en Genómica, Genética y Bioinformática, Ciudad

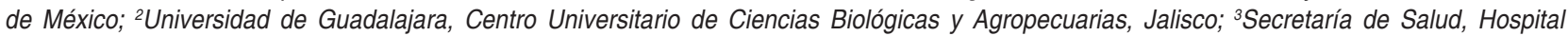 \\ Infantil de México "Federico Gómez", Laboratorio de Investigación en Biología del Desarrollo y Teratogénesis Experimental, Ciudad de México. \\ México
}

\section{Resumen}

La revisión por pares tradicional atraviesa por crecientes cuestionamientos, dado el aumento en el fraude científico detectado y la crisis de replicación que recientemente se ha presentado en la investigación biomédica. Investigadores, instituciones académicas y agencias de financiamiento activamente promueven el análisis del registro científico y se han desarrollado múltiples herramientas para lograrlo. Diferentes revistas biomédicas se fundaron con la revisión por pares pospublicación como característica; existen varias plataformas digitales que hacen posible este proceso. Asimismo, cada vez hay más revistas biomédicas que permiten comentar artículos publicados en sus sitios web, lo cual también es posible en repositorios de preimpresiones. Sumado a esto, las casas editoriales y los investigadores están usando ampliamente las redes sociales para la difusión y discusión de artículos, lo cual a veces culmina en refutaciones y retracciones.

PALABRAS CLAVE: Revisión por pares pospublicación. Redes sociales. Biomedicina.

\section{Post-publication peer review: another sort of quality control of the scientific record in biomedicine}

\begin{abstract}
Traditional peer review is undergoing increasing questioning, given the increase in scientific fraud detected and the replication crisis biomedical research is currently going through. Researchers, academic institutions, and research funding agencies actively promote scientific record analysis, and multiple tools have been developed to achieve this. Different biomedical journals were founded with post-publication peer review as a feature, and there are several digital platforms that make this process possible. In addition, an increasing number biomedical journals allow commenting on articles published on their websites, which is also possible in preprint repositories. Moreover, publishing houses and researchers are largely using social networks for the dissemination and discussion of articles, which sometimes culminates in refutations and retractions.
\end{abstract}

KEY WORDS: Post-publication peer review. Social networks. Biomedicine. 


\section{Introducción}

La revisión por pares (peer-review) es el proceso que permite a la comunidad de investigadores procurar la calidad del registro científico. En un artículo previo ${ }^{1}$ discutimos los mecanismos y los problemas más importantes que enfrenta en la actualidad el proceso de revisión por pares tradicional. En general, un panorama de alto volumen y bajo incentivo para los revisores ha condicionado un aumento en el material requerido para publicar un artículo, así como un incremento sustancial del tiempo para completar los procesos de evaluación, lo cual, en conjunto, ha potenciado el fraude científico. ${ }^{1}$

Una vez publicado, un artículo pasa a formar parte del registro científico, a menos que sea retraído por los autores o el editor de la revista donde fue difundido. Como una reacción a los problemas inherentes a la revisión por pares, en los últimos años hemos sido testigos de una diversificación en los medios que permiten la evaluación de un artículo después de su publicación, la cual se ha denominado revisión por pares pospublicación.

En vista de la llamada "crisis de replicación", 2 que ha llevado a sugerir que la mayor parte de la ciencia publicada es falsa, ${ }^{3}$ y de la progresiva reducción de recursos económicos disponibles, la necesidad del escrutinio del registro científico se ha vuelto más evidente que nunca para académicos, instituciones y agencias que financian la investigación.

Tradicionalmente, uno de los pocos medios para la evaluación de un artículo tras su publicación era la carta al editor. En este tipo de comunicaciones, uno o varios autores pueden expresar su reserva en cuanto al rigor, valor metodológico o validez de las conclusiones vertidas en un artículo. Sin embargo, este proceso suele ser lento y con frecuencia se encuentran grados variables de resistencia por parte de los editores de las revistas biomédicas, dado que potencialmente puede evidenciar defectos en la revisión y edición científica.

En las secciones siguientes, enumeramos los esfuerzos que en distintos frentes se llevan a cabo para hacer más rápida y eficiente la depuración del registro científico ya existente (Tabla 1).

\section{Revistas y plataformas con revisión por pares pospublicación}

A raíz de los problemas mencionados, inherentes al esquema clásico de publicación, diferentes entidades han creado revistas que, de inicio, funcionaron bajo el esquema de revisión por pares pospublicación. F1000Research, ${ }^{4}$ las revistas Copernicus, ${ }^{5}$ MedEdPublish ${ }^{6}$ y Sci ${ }^{7}$ son los ejemplos más emblemáticos de esta modalidad y que, adicionalmente, promueven el concepto de revisión por pares abierta, en la que todo el intercambio de información, los cuestionamientos y sus respuestas se hacen públicos en la medida en que se generan, junto con todas las versiones sometidas por los autores a lo largo del proceso.

El creciente volumen de literatura científica que se publica diariamente hace muy difícil para un lector interesado en un tema específico estar al tanto de todos los avances en su campo. Esto motivó la creación de una plataforma dedicada a la revisión por pares pospublicación, F1000Prime. ${ }^{8}$ El contenido de este recurso es aportado por paneles de reconocidos investigadores convocados por invitación, quienes periódicamente someten recomendaciones de artículos que a su juicio son de trascendencia para su campo de estudio y merecen especial atención. Pubpeer, ${ }^{9}$ por otro lado, es otro recurso que permite la discusión y el análisis, incluso anónimo, de obra publicada. Existen ejemplos de detección de fraude científico que llevaron a retracciones de artículos gracias a este recurso. ${ }^{10}$ Adicionalmente, ciertas plataformas, cuyo propósito principal es otro, permiten la revisión por pares pospublicación, entre ellas Researchgate,,$^{11}$ una red social de amplio uso entre los investigadores, en la cual los usuarios pueden comentar artículos que otros autores colocan en sus perfiles personales. Publons ${ }^{12}$ es una plataforma diseñada para construir un perfil del investigador, en la que se incorpora producción científica en términos de artículos, patentes, así como revisiones pospublicación de literatura generada por otros investigadores. Finalmente, The Winnower, ${ }^{13} \mathrm{RIO}$, PubPub y ScienceOpen ${ }^{14}$ son soluciones para todas las etapas del proceso editorial, que incluyen naturalmente funcionalidades para la revisión por pares pospublicación.

\section{Comentarios al calce de artículos en línea y repositorios de preimpresiones}

Actualmente, la inmensa mayoría de las revistas científicas mantiene páginas de Internet en las cuales es posible consultar tablas de contenido de los números publicados, además del título, resumen y, en ocasiones gratuitamente o previo pago, el contenido de 
Tabla 1. Direcciones en internet de los diferentes recursos para revisión por pares pospublicación mencionados en este artículo

\begin{tabular}{l|ll}
\hline Recurso & Dirección en internet & Descripción \\
\hline F1000Research & https://f1000research.com/ & Plataforma de publicación \\
\hline Copernicus & https://publications.copernicus.org/ & Publicación de revistas con RPPP \\
\hline MedEdPublish & https://www.mededpublish.org/ & Revista electrónica con la modalidad de RPPP \\
\hline Sci & https://www.mdpi.com/journal/sci & Revista electrónica con la modalidad de RPPP \\
\hline F1000Prime & https://f1000.com/ & Servicio de recomendación de artículos \\
\hline Pubpeer & https://pubpeer.com/ & Plataforma en línea para la RPPP \\
\hline Researchgate & https://www.researchgate.net/ & Red profesional para científicos e investigadores \\
\hline Publons & https://publons.com/ & Plataforma para construir perfiles de investigadores \\
\hline The Winnower & https://thewinnower.com/ & Plataforma de publicación académica en línea de acceso abierto que emplea RPPP \\
\hline RIO & https://riojournal.com/ & Plataforma editorial académica \\
\hline PubPub & https://www.pubpub.org/ & Sitio que apoya en cada paso el proceso de publicación \\
\hline ScienceOpen & https://www.scienceopen.com/ & Plataforma de libre acceso que ayuda a investigadores a publicar \\
\hline Arxiv & https://arxiv.org/ & Servicio de distribución gratuito y abierto de artículos académicos \\
\hline BioRxiv & https://www.biorxiv.org/ & Servicio gratuito en línea de archivo y distribución de preimpresiones inéditas \\
\hline RPPP = revisión por pares pospublicación. &
\end{tabular}

cada artículo. Cada vez con más frecuencia, al calce de cada artículo, se incluyen secciones en las cuales los usuarios pueden dejar comentarios a la publicación. Generalmente, los comentarios pueden ser escritos previo inicio de sesión en el sistema de la revista o su casa editorial o bien en Twitter, Facebook u otras redes sociales. Dependiendo de la revista, estos comentarios son desplegados inmediatamente o previa aprobación por un moderador con el fin de limitar el uso de lenguaje ofensivo o inapropiado.

En una comunicación previa analizamos la importancia y las implicaciones del modelo de las preimpresiones en biomedicina. ${ }^{1}$ En este artículo, esta modalidad requiere también mención, en el sentido de que en los repositorios de preimpresiones (es decir, sitios de Internet donde se almacenan y se distribuyen esas publicaciones) como Arxiv o BioR $\chi$ iv, entre otros, se propicia una forma de revisión por pares pospublicación en la forma de comentarios al calce de cada preimpresión, similar al descrito en el párrafo anterior.

\section{El impacto de las redes sociales}

Los grandes avances en telecomunicaciones experimentados en las últimas décadas han propiciado el florecimiento explosivo de diversas redes sociales.
Las diferentes casas editoriales no han permanecido al margen de este fenómeno y actualmente no es inusual encontrar que revistas biomédicas administran cuentas en diferentes redes, especialmente en Twitter, Facebook e Instagram. Esta tendencia, un descendiente directo de las listas de contenidos que periódicamente envían las revistas científicas vía correo electrónico, ha agilizado enormemente la difusión de nuevos artículos entre los potenciales lectores. Adicionalmente, dado su amplísimo uso, gran parte de la discusión actual sobre publicaciones científicas ocurre al amparo de las redes sociales. Diversos estudios encuentran una correlación positiva entre tuits relativos a una publicación y las citas que obtiene..$^{15-17}$

La potencia de las redes sociales como medios para la revisión pospublicación ha sido evidenciada: comentarios en Twitter desencadenaron procesos que culminaron en refutaciones y retracciones de artículos en la prestigiosa revista Science. ${ }^{18}$

\section{Conclusiones y consideraciones finales}

Las motivaciones para hacer una revisión por pares pospublicación de un artículo pueden ser variadas, por ejemplo, en la medida en que se dispone de 
nuevas tecnologías, los hallazgos previos pueden ser interpretados en distintas formas: se generan nuevas observaciones que desacreditan su metodología o resultados, o la incapacidad para reproducir sus experimentos o resultados. Otra motivación es la selección de artículos con especial relevancia para el avance de un campo específico, resaltándolo sobre otros en apariencia similares.

Gracias al uso extendido y prevalente de Internet por los investigadores biomédicos y los consumidores de la literatura que generan, estamos asistiendo a la diversificación y al crecimiento orgánicos de recursos diseñados para crear, difundir y evaluar ciencia. Solo podemos esperar que esta enorme variedad de recursos, completa o parcialmente dedicados a la revisión por pares pospublicación, se incremente en el futuro cercano; su clasificación y descripción pueden ser confusas si no se considera cómo se originaron y cómo se transforman, ya que, parafraseando a Theodosius Dobzhansky, nada en biología tiene sentido, excepto a la luz de la evolución. ${ }^{19}$

\section{Conflicto de intereses}

Los autores declaran no tener conflicto de intereses alguno.

\section{Fuentes de financiamiento}

Los autores no recibieron patrocinio para llevar a cabo este artículo.

\section{Responsabilidades éticas}

Protección de personas y animales. Los autores declaran que para esta investigación no se realizaron experimentos en seres humanos ni en animales.
Confidencialidad de los datos. Los autores declaran que en este artículo no aparecen datos de pacientes.

Derecho a la privacidad y consentimiento informado. Los autores declaran que en este artículo no aparecen datos de pacientes.

\section{Bibliografía}

1. Aquino-Jarquín G, Valencia-Reyes JM, Silva-Carmona A, Granados-Riverón JT. Preimpresiones en biomedicina: ¿alternativa o complemento al modelo tradicional de publicación? Gac Med Mex. 2018;154:87-91.

2. Baker M. 1,500 scientists lift the lid on reproducibility. Nature. 2016;533:452-454.

3. Ioannidis JPA. Why most published research findings are false. PLoS Med. 2005;2:e124-e124.

4. Kirkham J, Moher D. Who and why do researchers opt to publish in post-publication peer review platforms? - findings from a review and survey of F1000 Research. F1000Res. 2018;7:920.

5. Schmidt B, Ross-Hellauer T, van Edig X, Moylan EC. Ten considerations for open peer review. F1000Res. 2018;7:969.

6. MedEdPublish. Med Teach. 2015;37:794-796.

7. Rittman M, Vázquez F. Sci-An open access journal with post-publication peer review. Sci. 2019;1:1

8. Bornmann L, Haunschild R. Do altmetrics correlate with the quality of papers? A large-scale empirical study based on F1000Prime data. PLoS One. 2018;13:e0197133-e0197133.

9. Teixeira-da Silva JA, Al-Khatib A, Dobránszki J. Fortifying the corrective nature of post-publication peer review: identifying weaknesses, use of journal clubs, and rewarding conscientious behavior. Sci Eng Ethics. 2017;23:1213-1226.

10. Koppers L, Wormer H, Ickstadt K. Towards a systematic screening tool for quality assurance and semiautomatic fraud detection for images in the life sciences. Sci Eng Ethics. 2017;23:1113-1128.

11. Meier A, Tunger D. Survey on opinions and usage patterns for the ResearchGate platform. PLoS One. 2018;13:e0204945.

12. Swiontkowski M. Publons: the next step in reviewer recognition. J Bone Joint Surg Am. 2019;101:1137.

13. Tattersall A. For what it's worth-the open peer review landscape. Online Inf Rev. 2015;39:649-663.

14. Tennant JP, Dugan JM, Graziotin D, Jacques DC, Waldner F, Mietchen D, et al. A multi-disciplinary perspective on emergent and future innovations in peer review. F1000Res. 2017;6:1151.

15. Eysenbach G. Can tweets predict citations? Metrics of social impact based on Twitter and correlation with traditional metrics of scientific impact. J Med Internet Res. 2011;13:e123.

16. Shuai $X$, Pepe A, Bollen J. How the scientific community reacts to newly submitted preprints: article downloads, Twitter mentions, and citations. PLoS One. 2012;7:e47523.

17. Chiang AL, Rabinowitz LG, Alakbarli J, Chan WW. Tu1108 social media exposure is independently associated with increased citations of publications in gastroenterology. Gastroenterology. 2016;150:S845.

18. Yeo SK, Liang X, Brossard D, Rose KM, Korzekwa K, Xenos MA, et al. The case of \#arseniclife: blogs and Twitter in informal peer review. Public Underst Sci. 2017;26:937-952.

19. Dobzhansky T. Nothing in biology makes sense except in the light of evolution. Am Biol Teach. 1973;35:125-129. 